\title{
Interférences
}

Ars scribendi

$10 \mid 2018$

Varia

\section{Gallien, Claude le Gothique, Aurélien et le bon empereur dans les Caesares d'Aurelius Victor}

\section{Hermann Amon}

\section{(2) OpenEdition \\ Journals}

Édition électronique

URL : http://journals.openedition.org/interferences/5968

DOI : 10.4000/interferences.5968

ISSN : 1777-5485

Éditeur

HiSoMA - Histoire et sources des Mondes antiques

Référence électronique

Hermann Amon, «Gallien, Claude le Gothique, Aurélien et le bon empereur dans les Caesares d'Aurelius Victor », Interférences [En ligne], 10 | 2018, mis en ligne le 09 février 2018, consulté le 15 septembre 2020. URL : http://journals.openedition.org/interferences/5968 ; DOI : https://doi.org/10.4000/ interferences. 5968

Ce document a été généré automatiquement le 15 septembre 2020.

Tous droits réservés 


\title{
Gallien, Claude le Gothique, Aurélien et le bon empereur dans les Caesares d'Aurelius Victor
}

\author{
Hermann Amon
}

\section{Introduction}

1 La question de l'empereur idéal occupe une place essentielle dans l'historiographie impériale ${ }^{1}$. Plusieurs auteurs romains, particulièrement ceux de langue grecque tels Dion Cassius, Hérodien ou encore le Pseudo Aelius Aristide, ont esquissé dans leurs ouvrages l'archétype de l'empereur idéal ${ }^{2}$, qui se résume en la possession des vertus impériales ${ }^{3}$. En effet, ces vertus sont un ensemble de qualités et d'attributs divins qui, octroyés par les dieux à l'empereur, soulignent le caractère exceptionnel de sa personne ${ }^{4}$. En outre, elles contribuent à la propagande idéologique du régime puisqu'elles permettent de projeter une image sacro-sainte de l'empereur auprès de l'opinion publique à travers les monnaies, les inscriptions, les panégyriques, les décrets et les biographies ${ }^{5}$. Au nombre de ces vertus impériales figurent les vertus augustéennes que sont la virtus, la clementia, la iustitia et la pietas ${ }^{6}$. Il s'agit des quatre vertus inscrites sur le bouclier d'or offert par le Sénat à l'empereur Auguste en reconnaissance de son action exceptionnelle dans la pacification de l'Empire et dans le rétablissement des institutions ${ }^{7}$. S'ajoute un nombre non exhaustif de personnifications et de qualités divines tirées de l'environnement politique et religieux romain ${ }^{8}$. Contrairement à ce qu'avait suggéré M. Charlesworth en se basant sur le bouclier d'or reçu du Sénat par Auguste 9 , ces vertus impériales n'ont jamais été inscrites dans un canon. Elles sont bien au contraire le reflet de la constante évolution de l'idéologie impériale, qui elle-même dépend du contexte politique et militaire de l'Empire ${ }^{10}$. Aussi, puisqu'elles participent à la propagande politique, l'empereur pouvait choisir de mettre l'accent sur l'une ou l'autre des vertus ou qualités, en fonction des circonstances du moment ${ }^{11}$. Au-delà donc de cette image projetée par l'empereur s'ajoute celle transmise 
par les auteurs, contemporains ou non des événements. C'est cette seconde image qui est au centre de notre analyse.

$\mathrm{Au}$ III ${ }^{\mathrm{e}}$ siècle, les guerres aux frontières et leurs conséquences ont un impact majeur sur l'image de l'empereur et, par voie de conséquence, sur le modèle du bon empereur. Dans ce contexte, quel est le modèle du bon empereur à cette époque? Quels sont les qualités et attributs qui le caractérisent? L'objectif de cet article est de répondre à ces questions à partir des biographies des empereurs Gallien, Claude le Gothique et Aurélien dans les Caesares d'Aurelius Victor ${ }^{12}$. En effet, malgré les critiques sur les erreurs factuelles de son récit, ses commentaires moralisants et sa négligence vis-à-vis de la chronologie ${ }^{13}$, le Liber de Caesaribus d'Aurelius Victor demeure une source importante pour la compréhension de la période charnière du III ${ }^{e}$ siècle de l'Empire. Audelà de la biographie des empereurs, Victor décrit l'évolution de la vie politique romaine et fournit de précieux détails sur la construction idéologique des empereurs. Dans cette perspective, les vertus impériales et la définition du bon empereur occupent une place essentielle dans son récit puisqu'elles conditionnent et facilitent les succès ultérieurs des princes et la prospérité ou la ruine de l'État. C'est ce qu'il confirme de manière explicite (Aurelius Victor, Liber de Caesaribus, XXXV, 14) :

adhuc virtutibus principum res attolli facile vel afflictas, easque firmiores praeceps vitiis

dari

"aussi, que les vertus des princes relèvent facilement les empires même abattus,

tandis que leurs vices précipitent la ruine des États les plus solidement affermis ».

En outre, les biographies de Gallien, Claude le Gothique et Aurélien permettent à Victor, et à travers lui à l'aristocratie sénatoriale des $\mathrm{IV}^{\mathrm{e}}$ et $\mathrm{V}^{\mathrm{e}}$ siècles, de proposer une figure archétypale de l'optimus et du pessimus princeps pour le III $^{\mathrm{e}}$ siècle, établissant le bon et le mauvais exemple à suivre. En effet, au cours de leurs règnes respectifs (260-278), l'Empire passe d'une période de profonde instabilité à un début de rétablissement, caractérisé entre autres par la fin des sécessions de la Gaule et de Palmyre. Cette évolution de la situation aux frontières affecte nécessairement la vision du bon et du mauvais empereur. Toutefois, il ne s'agira pas de faire une étude des différents règnes mais plutôt, à partir d'une analyse dynamique et thématique, de présenter les qualités du bon empereur pour le $\mathrm{III}^{\mathrm{e}}$ siècle. Nous discuterons dans une première partie des qualités morales et, dans une seconde, des qualités militaires essentielles pour cette période de crise.

\section{Les qualités morales du bon empereur}

Les vertus morales du prince sont un sujet important des biographies de Victor puisqu'elles servent de fondation à l'excellence de l'activité politique et militaire du bon empereur. Ainsi Trajan, l'optimus princeps par excellence, est qualifié de aequus, clemens, patientissimus atque in amicos perfidelis ${ }^{14}$, "juste, clément, le plus patient des hommes, et surtout le plus fidèle des amis ", pour souligner sa sobriété et la grandeur de ses qualités morales. Au-delà de leur importance dans l'exercice de l'activité politique, ces vertus mettent en lumière l'exemplarité du caractère de l'empereur. C'est ce que confirme Victor à travers cette anecdote sur l'empereur Trajan (Aurelius Victor, Liber de Caesaribus, XIII, 9) : 
usque eo innocentiae fidens, uti praefectum praetorio Suburanum nomine, cum insigne potestatia, uti mos erat, pugionem daret, crebro monuerit : "Tibi istum ad munimentum mei committo, si recte agam ; sin aliter, in me magis "

« il avait tant de confiance en la vertu qu'en présentant, selon la coutume, au préfet du prétoire Suburanus un poignard comme insigne de son pouvoir, il lui donna le conseil suivant: "Je te le confie pour me défendre, si je me conduis bien; sinon serst'en plutôt contre moi." "

Il utilise des qualificatifs similaires lorsqu'il évoque Marc Aurèle, l'autre exemple du bon empereur (Aurelius Victor, Liber de Caesaribus, XVI, 9) :

tantumque Marco sapientiae lenitudinis innocentiae ac litterarum fuisse

« et que Marc Aurèle était homme si sage si doux, si probe et si cultivé ».

Cette importance des valeurs morales pour le bon empereur reste valable pour le $\mathrm{III}^{\mathrm{e}}$ siècle. Claude le Gothique est ainsi décrit comme étant un homme travailleur, juste et dévoué à l'État (Aurelius Victor, Liber de Caesaribus, XXXIV, 1) :

viri laborum patientis aequique ac prorsus dediti reipublicae, quippe ut longo intervallo Deciorum morem renovaverit

« [Claude] était un homme dur à la fatigue, juste et totalement dévoué à l'État ».

Victor poursuit pour ajouter :

Quippe ut longo interuallo Deciorum morem renouauerit

« Lui qui longtemps après la mort des Decii fit revivre leur caractère ».

La référence aux Decii, c'est-à-dire à Dèce et à ses fils Hostilien et Volusien ${ }^{15}$, est révélatrice des valeurs morales que doit porter le bon empereur. En effet, l'empereur Dèce s'était résolument engagé dans la protection des frontières de l'Empire face aux attaques germaniques ${ }^{16}$. En outre, il avait fondé le succès de sa stratégie militaire sur un raffermissement du culte des dieux infernaux romains ${ }^{17}$. Ce parallèle entre Claude le Gothique et les Decii souligne indubitablement l'importance de la fidélité à l'État et aux divinités tutélaires de Rome dans la qualification du bon empereur. La crise militaire aux frontières exigeait de l'empereur un engagement sans faille pour l'État et ses symboles. Ailleurs, Victor souligne que Claude se soucie de la protection de ses concitoyens $^{18}$. Cette compassion de l'empereur pour ses concitoyens constitue une autre de ses qualités morales ${ }^{19}$.

Dans le même ordre d'idées, Aurélien est qualifié, après sa mort, de grand homme pour son austérité et pour l'intégrité de son caractère (Aurelius Victor, Liber de Caesaribus, $\mathrm{XXXV}, 12)$ :

Tantum ille vir severitate atque incorruptis artibus potuit

«Le grand homme que fut Aurélien, par son austérité et par l'intégrité de son caractère ».

Pour Victor, cette severitas d'Aurélien ne doit pas être rattachée au terme "crudelitas ", mais plutôt à une haine de la corruption ${ }^{20}$. En effet, à la différence d'Eutrope et de l' Histoire Auguste, Victor assimile cette severitas à une rigueur et une droiture morale ${ }^{21}$. C'est justement cette intégrité morale d'Aurélien qui se manifeste par les actions de moralisation de la vie publique qu'il engage sous son règne ${ }^{22}$. Il avait mis fin à la révolte des monétaires et à l'activité des quadruplatores ${ }^{23}$.

Victor amplifie les qualités d'Aurélien lorsqu'il met en exergue sa générosité et le présente comme le protecteur des démunis. Aurélien avait effectué diverses distributions de vivres à la plèbe et introduit la consommation de la viande de porc ${ }^{24}$. En effet, la générosité de l'empereur est fondamentale dans la propagande impériale puisqu'elle réfère à la notion de liberalitas ${ }^{25}$, qui souligne l'obligation morale des 
empereurs de fournir à leurs sujets les ressources nécessaires à leur survie. L'empereur Auguste en fut l'un des promoteurs et, à sa suite, plusieurs empereurs en feront une vertu essentielle de leur règne ${ }^{26}$.

Chez Aurelius Victor comme dans l'Histoire Auguste et chez Eutrope, les qualités morales de Claude et Aurélien sont mises en opposition avec les traits de Gallien, le contreexemple du bon empereur ${ }^{27}$. On retrouve d'ailleurs dans les biographies de Claude le Gothique et d'Aurélien des références constantes au règne de Gallien pour souligner ce contraste. Aussi, dans la longue biographie qu'il lui consacre, Victor le qualifie tout d'abord d'efféminées. L'auteur de l'Histoire Auguste utilise le terme de remissius, «mollesse $\mathrm{e}^{29}$ ». Plus loin, celui-ci ajoute que son règne était inférieur à celui des femmes, en le comparant à Zénobie ${ }^{30}$. Cette caractéristique n'est pas nouvelle dans le langage politique romain, elle est constamment utilisée pour railler et ternir l'image d'un adversaire $^{31}$. Elle symbolise non seulement la lâcheté et la couardise, mais renvoie aussi à certains traits de caractère féminins ${ }^{32}$. D'ailleurs, c'est le qualificatif par excellence des mauvais empereurs ${ }^{33}$. Victor poursuit l'exposition du contre-exemple lorsqu'il dénonce l'insouciance et la frivolité de Gallien qui se livre aux plaisirs en compagnie de son épouse et d'une princesse étrangère (Aurelius Victor, Liber de Caesaribus, XXXIII, 4-7) :

Ita quasi ventis undique saevientibus parvis maxima ima summis orbe toto miscebantur. Simulque Romam pestilentia grassabatur, quae saepe curis gravioribus atque animi desperatione oritur. Inter haec ipse popinas ganeasque obiens lenonum ac vinariorum amicitiis haerebat, expositus Saloninae coniugi atque amori flagitioso filiae Attali Germanorum regis, Pipae nomine.

«Ainsi l'on eût dit que la tempête se déchaînait de toutes parts, et que, dans le monde entier, petitesse et grandeur, abaissement et élévation étaient mêlés et confondus. Rome était assaillie par de la peste, qui souvent surgit lors de graves inquiétudes. Au milieu de tant de fléaux, Gallien, l'empereur, hantait les tavernes et les lieux de débauche, s'attachait aux souteneurs et aux ivrognes, s'abandonnant à sa femme Salonine ou à Pipa, la fille d'Attale, roi des Germains. »

En plus de l'insouciance, le pessimus princeps se caractérise par une absence de moderatio ${ }^{34}$. Il s'agit explicitement ici du manque de contrôle sur ses désirs et ses passions. Le pessimus princeps se trouve ainsi plus préoccupé à assouvir ses plaisirs, qu'ils soient sexuels ou alimentaires, qu'à accomplir son devoir envers l'État. Par son insouciance et la lubricité de son caractère, Gallien se range dans cette catégorie. Il préfère satisfaire ses plaisirs plutôt que répondre à la peste qui frappe Rome. Cette insouciance de Gallien est largement élaborée par l'Histoire Auguste, lorsqu'elle évoque entre autres l'inaction de Gallien après la capture de son père Valérien et la multiplication des coups d'État ${ }^{35}$.

Le bon empereur est ainsi celui qui fait preuve de modération sur le plan des mœurs, protège ses concitoyens, et reste fidèle aux traditions romaines. En agissant de la sorte, il obtient l'assistance des dieux pour la victoire militaire contre les ennemis de l'Empire. $\mathrm{Au}$ III ${ }^{\mathrm{e}}$ siècle, cette corrélation entre qualités morales et succès militaires est déterminante dans la définition du bon empereur. 


\section{Les qualités militaires du bon empereur}

Les qualités morales du prince sont indissociables de sa clairvoyance politique et de ses aptitudes militaires. Par clairvoyance politique, il faut entendre la capacité, pour l'empereur, de maintenir une excellente relation avec les forces politiques que sont le Sénat et l'armée, dans le but de garantir un régime stable et durable. Ainsi, tandis que Claude accède au pouvoir avec le soutien du Sénat ${ }^{36}$, Gallien, le mauvais exemple, écarte les sénateurs de la gestion de l'armée et des fonctions militaires ${ }^{37}$ (Aurelius Victor, Liber de Caesaribus, XXXIII, 33-35):

Et patres quidem praeter commune Romani malum orbis stimulabat proprii ordinis contumelia, quia primus ipse metu socordiae suae, ne imperium ad optimos nobilium transferretur, senatum militia vetuit et adire exercitum.

"Les sénateurs, outre les maux communs au monde romain, étaient ulcérés de l'affront subi par leur ordre. En effet, Gallien, le premier redoutant sa propre incurie et craignant de voir le pouvoir être transféré aux plus illustres de la noblesse, leur interdit le service militaire, et même l'accès de l'armée. »

Selon Victor, cette mise à l'écart avait pour objectif de réduire les coups d'État. Les proches de Gallien seront d'ailleurs victimes de représailles après sa mort ${ }^{38}$. Sur le plan strictement militaire, le bon empereur n'est pas seulement celui qui marche à la tête des soldats, mais aussi et surtout celui qui remporte des victoires grâce à sa bravoure et à son intelligence stratégique. Dans cette perspective, Claude est présenté comme un stratège militaire ayant remporté plusieurs victoires contre les Germains (Aurelius Victor, Liber de Caesaribus, XXXIV, 5) :

Ita nullo exercitus detrimento fusi barbari summotique

«Ainsi sans aucun dommage pour l'armée, les barbares furent mis en déroute et chassés de l'Empire ».

Victor fait référence aux victoires de Claude obtenues en 269 contre les Goths à Naissus et contre les Alamans sur les rives du lac de Garde ${ }^{39}$. Ce sont ces victoires qui lui valurent le titre de Gothicus Maximus ${ }^{40}$.

Toujours dans la dynamique du bon et du mauvais exemple, le contraste entre Claude et Aurélien avec Gallien s'accentue. Quand Aurélien construit une enceinte pour protéger Rome et ses habitants ${ }^{41}$, Gallien distille de fausses informations sur la situation aux frontières et préfère s'adonner à des plaisirs en organisant des jeux (Aurelius Victor, Liber de Caesaribus, XXXIII, 15-16) :

At Romae Gallienus pacata omnia ignaris publici mali improbe suadebat, crebro etiam, uti rebus ex voluntate gestis solet, ludos ac festa triumphorum, quo promptius simulata confirmarentur, exercens.

"À Rome cependant, Gallien faisait croire faussement à ceux qui ignoraient les malheurs publics que tout l'Empire était en paix; souvent même, selon l'usage de ceux dont le seul but est de tromper, il faisait célébrer des jeux et des fêtes triomphales, pour mieux confirmer la réalité de ses mensonges. »

Submergé par ses passions, Gallien n'arrive pas à protéger l'Empire qui se disloque sous ses yeux ${ }^{42}$ (Aurelius Victor, Liber de Caesaribus, XXXIII, 3-6) :

His prospere ac supra vota cedentibus more hominum secundis solutior rem Romanam quasi naufragio dedit

« Après de tels succès, qui allaient au-delà de ses espérances, amolli, comme cela est naturel aux hommes, par la prospérité, il laissa pour ainsi dire sombrer la puissance romaine ». 
19 Aurélien est en revanche respecté des soldats ${ }^{43}$, et montré en exemple pour ses succès militaires contre les Perses (Aurelius Victor, Liber de Caesaribus, XXXV, 1) :

Ceterum Aurelianus successit tanto vehementior confestim, quasi belli reliquiae superessent, in Persas progressus est.

"Aurélien, dont une réussite aussi grande avait stimulé l'ardeur martiale, pour en finir, en quelque sorte, avec les restes de la guerre, marche aussitôt contre les Perses. »

20 Victor évoque ici le succès de la reconquête de Palmyre et une brève campagne contre les Perses ${ }^{44}$ à l'issue de laquelle Aurélien reçut les titres de Persicus Maximus et de Particus Maximus ${ }^{45}$. Plus loin, il renchérit sur l'intelligence stratégique d'Aurélien lorsqu'il évoque son action dans la fin de la sécession gauloise (Aurelius Victor, Liber de Caesaribus, XXXV, 3-6) :

Simul, Germanis Gallia dimotis, Tetrici, de quo supra memoravimus, caesae legiones proditore ipso duce. Namque Tetricus, cum Faustini praesidis dolo, corruptis militibus plerumque peteretur, Aureliani per litteras praesidium imploraverat eique adventanti producta ad speciem acie inter pugnam se dedit. Ita, uti rectore nullo solet, turbati ordines oppressi sunt

« En même temps, après avoir chassé les Germains de Gaule, il tailla en pièces les légions de Tetricus, dont nous avons parlé plus haut, grâce à la trahison de leur chef lui-même. En effet, Tetricus, souvent en butte aux intrigues du gouverneur Faustinus qui avait corrompu ses soldats, avait, par une lettre, imploré l'aide d'Aurélien, et quand l'empereur vint à sa rencontre, il fit, pour faire illusion, avancer sa ligne de bataille, puis se livra au milieu du combat. Ainsi, comme cela se produit habituellement quand il n'y a plus de commandement, le désordre se mit dans les rangs et l'armée fut écrasée ».

\section{Conclusion}

21 Le bon empereur se distingue par ses vertus, lesquelles soulignent le caractère exceptionnel de sa personne et légitiment son autorité. Deux groupes de qualités ou vertus sont essentiels dans la définition du bon empereur pour le $\mathrm{III}^{\mathrm{e}}$ siècle. Il s'agit des qualités morales qui servent de fondation aux qualités militaires. Sur la base des trois biographies analysées dans les Caesares, le bon empereur se caractérise par la sobriété de ses mœurs, par sa fidélité aux symboles de l'État romain et par son engagement pour la protection de Rome et de ses habitants. De plus, il se singularise par ses compétences militaires et son respect de l'autorité du Sénat. Pour Victor, les vertus morales et les qualités militaires de l'empereur garantissent ses succès de chef des armées dans une période de crise. Par conséquent, le redressement de la situation politique amorcé sous Claude le Gothique et amplifié sous Aurélien est le résultat de leurs qualités morales et militaires. En revanche, la décadence romaine sous Gallien est directement reliée à son absence de vertus et sa défiance du Sénat. 


\section{BIBLIOGRAPHIE}

\section{Textes anciens}

Aurelius Victor, Livre des Césars, texte établi et trad. par P. DUFRAIGNE, CUF, Paris, Les Belles

Lettres, 1975.

Eutrope, Abrégé d'histoire romaine, texte établi et trad. par J. HELLEGOUARC'H, CUF, Paris, Les Belles Lettres, 1999.

Hérodien, Histoire des empereurs romains de Marc-Aurèle à Gordien III (180 apr. J.-C. - 238 apr. J.-C.), trad. et commenté par D. ROQUES, La Roue à livres, Paris, Les Belles Lettres, 1990.

Histoire Auguste. Les Empereurs romains des $I^{e}{ }^{e}$ et III ${ }^{e}$ siècles, trad. du latin par A. [et J.] CHASTAGNOL ; éd. établie par A. CHASTAGNOL, Paris, R. Laffont, 1994.

Suétone, Vie des douze Césars. II, Tibère, Caligula, Claude, Néron, texte établi et trad. par H. AILLOUD, CUF, Paris, Les Belles Lettres.

\section{Études modernes}

AALDERS G. J. D. 1986, « Cassius Dio and the Greek World », Mnemosyne 39, 3, p. 282-304.

ALLARD V. 2006, « La crudelitas d'Aurélien », in M.-H. QUET (éd.), La “crise” de l'Empire romain de Marc Aurèle à Constantin, Paris, Presses de l'Université Paris-Sorbonne, p. 173-184.

BARNES T. D. 1978, The Sources of the Historia Augusta, Bruxelles, Latomus.

BIRD H. W. 1984, Sextus Aurelius Victor. A Historiographical Study, ARCA Classical and Medieval Texts, Papers and Monographs 14, Liverpool, F. Cairns.

BURGESS R. W. 1995, « On the Date of the Kaisergeschichte », CPh 90, p. 111-128.

CHARLESWORTH M. P. 1937, « The Virtues of a Roman Emperor: Propaganda and the Creation of Belief », PBA 23, p. 105-133.

CHRISTOL M. 2006, L'Empire romain du III siècle. Histoire politique (de 192, mort de Commode, à 325, concile de Nicée), $2^{e}$ éd., revue et augm., Paris, Éd. Errance.

CLASSEN C. J. 1991, «Virtutes Imperatoriae », Arctos 25, p. 17-39.

CORBEILL A. 1996, Controlling Laughter: Political Humor in the Late Roman Republic, Princeton, Princeton University Press.

COSME P. 2007, « À propos de l'Édit de Gallien », in O. HEKSTER, G. DE KLEIJN, D. SLOOTJES (ed.), Crises and the Roman Empire. Proceedings of the Seventh Workshop of the International Network Impact of Empire, Nijmegen, June 20-24, 2006, Leyde, Brill, p. 97-109.

DE BLOIS 1994, "Traditional Virtues and New Spiritual Qualities in Third Century Views of Empire, Emperorship and Practical Politics », Mnemosyne 47, 2, p. 166-176.

- 1998, « Emperor and Empire in the Works of Greek-Speaking Authors of the Third Century AD », ANRW, II, 34, 4, p. 3391-3443. 
- 2003, «The Perception of Roman Imperial Authority in Herodian's Work », in L. DE BLOIS, P. ERDKAMP, O. HEKSTER, G. DE KLEIJN, S. MOLS (ed.), The Representation and Perception of Roman Imperial Power. Proceedings of the Third Workshop of the International Network Impact of Empire, Roman Empire, c. 200 B.C.-A.D. 476, Netherlands Institute in Rome, March 20-23, 2002, Impact of Empire, Roman Empire 3, Amsterdam, J. C. Gieben, p. 148-153.

DEMOUGEOT É. 1969, La formation de l'Europe et les invasions barbares. I, Des origines germaniques à l'avènement de Dioclétien, Collection historique, Paris, Aubier.

EARL D. C. 1967, The Moral and Political Tradition of Rome, Ithaca, Thames and Hudson.

EDWARDS C. 2002, The Politics of Immorality in Ancient Rome, Cambridge, Cambridge University Press.

FEARS J. R. 1981, « The Cult of Virtues and Roman Imperial Ideology », ANRW, XI, 17, 2, p. 827-948.

FREYBURGER-GALLAND M.-L. 1997, Aspects du vocabulaire politique et institutionnel de Dion Cassius, Études d'archéologie et d'histoire ancienne, Paris, De Boccard.

GAGÉ J. 1932, « Un thème de l'art impérial romain : la victoire d'Auguste », Mélanges d'archéologie et d'histoire 49, p. 61-92.

GANGLOFF A. 2009, «Le sophiste Dion de Prusse, le bon roi et l'empereur », RH 649, 1, p. 3-38.

- 2011, « Le princeps et le bon roi selon Homère », in S. BENOIST, A. DAGUET-GAGEY, C. HOËT-VAN CAUWENBERGHE (éd.), Figures d'empire, fragments de mémoire. Pouvoirs et identités dans le monde romain impérial (II s. av. n. è. - VI $I^{e}$ s. de n. è.), Archaiologia, Villeneuve d'Ascq, Presses universitaires du Septentrion, p. 105-122.

HARRIS W. V. (ed.) 2008, Aelius Aristides between Greece, Rome, and the Gods, Columbia Studies in the Classical Tradition 33, Leyde, Brill.

HEKSTER O. J. 2007, «Fighting for Rome: The Emperor as a Military Leader », in L. DE BLOIS, E. LO CASCIO (ed.), The Impact of the Roman Army. Economic, Social, Political, Religious, and Cultural Aspects. Proceedings of the Sixth Workshop ofthe International Network Impact of Empire (Roman Empire, 200 BCAD 476), Capri, March 29 - April 2, 2005, Leyde, Brill, p. 91-105.

KEMEZIS A. M. 2014, Greek Narratives of the Roman Empire under the Severans: Cassius Dio, Philostratus and Herodian, Greek Culture in the Roman World, Cambridge, Cambridge University Press.

KIENAST D. 1990, Römische Kaisertabelle. Gundzüge einer römischen Kaiserchronologie, Darmstadt, Wissenschaftliche Buchgesellschaft.

KLOFT H. 1970, Liberalitas Principis. Herkunft und Bedeutung. Studien zur Prinzipatsideologie, Kölner Historische Abhandlungen 18, Cologne, Böhlau.

KNIPFING J. R. 1923, « The Libelli of the Decian Persecution », HThR 16, p. 345-390.

LE BOHEC Y. 2004, «Gallien et l'encadrement sénatorial de l'armée », Revue des études militaires anciennes 1, p. 123-132.

LICHоскA B. 1974, Justitia sur les monnaies impériales romaines, Travaux au Centre d'archéologie méditerranéenne de l'Académie polonaise des sciences 15, Varsovie, PWN, Éditions scientifiques de Pologne.

LORIOT X. 1990, « Un sénateur illyrien élevé à la pourpre : Trajan Dèce », in E. FRÉZOULS, H. JOUFFROY (éd.), Les empereurs illyriens. Actes du colloque de Strasbourg, 11-13 octobre 1990, organisé par le Centre de recherche sur l'Europe centrale et sud-orientale, Contributions et travaux de l'Institut d'histoire romaine 8, Strasbourg, AECR, p. 43-55. 
MANDERS E. 2007, « Mapping the Representation of Roman Imperial Power in Times of Crisis », in O. HEKSTER, G. DE KLEIJN, D. SLOOTJES (éd.), Crises and the Roman Empire. Proceedings of the Seventh Workshop of the International Network Impact of Empire, Nijmegen, June 20-24, 2006, Leyde, Brill, p. 275-290.

MARKHOWSKI H. 1936, « De quattuor virtutibus Augusti in clupeo aureo ei dato scriptis », Eos 37, p. 109-128.

MILLAR F. 1964, A Study of Cassius Dio, Oxford, Clarendon Press.

MOLIN M. 2006, «Seueritas, une vertu politique romaine en échec au III siècle », in M.-H. QUET (éd.), La "crise" de l'Empire romain de Marc Aurèle à Constantin, Paris, Presses de l'Université ParisSorbonne, p. 185-210.

NAKAGAWA A. 2002, «L'imperatore Tiberio e la virtù della moderatio », Acme 55, 3, p. 226-228.

NOREÑA C. F. 2001, «The Communication of the Emperor's Virtues », JRS 91, p. 146-168.

OLIVER J. H. 1980, « The Ruling Power. A Study of the Roman Empire in the Second Century after Christ through the Roman Oration of Aelius Aristides », TAPhA 43, 4, p. 871-1003.

PFAFF-REYDELLET M. 2003, « Les vertus impériales et leur rôle dans la divination du prince selon Wissowa », $A R G$ 5, 1, p. 80-100.

POTTER D. S. 1999, « Political Theory in the Senatus Consultum Pisonianum », AJPh 120, 1, p. 65-88.

RIVES J. B. 1999 « The Decree of Decius and the Religion of Empire », JRS 89, p. 135-154.

ROLLER M. 2001, « Ethics for the Principate: Seneca, Stoicism, and Traditional Roman Morality », in Id., Constructing Autocracy. Aristocrats and Emperors in Julio-Claudian Rome, Princeton, Princeton University Press, p. 64-126.

SALISBURY F. S., MATTINGLY H. 1924, « The Reign of Trajan Decius », JRS 14, p. 1-23.

SEAGER R. 1984, « Some Imperial Virtues in the Latin Prose Panegyrics: The Demands of Propaganda and the Dynamics of Literary Composition ", in Papers of the Liverpool Latin Seminar, IV, Liverpool, F. Cairns, p. 129-165.

STERTZ S. A. 1977, « Marcus Aurelius as Ideal Emperor in Late-Antique Greek Thought », CW 70, p. $433-439$.

STONE POTTER D. 1999, « Political Theory in the senatus consultum pisonianum », AJPh 120, p. 65-88. тімотну T. D. 1970, « The Lost Kaisergeschichte », in Bonner Historia-Augusta-Colloquium 1968-1969, Bonn, R. Habelt, p. 22-23.

- 1978, The Sources of the Historia Augusta, Coll. Latomus 155, Bruxelles, Latomus.

WALLACE-HADRILl A. 1981, « The Emperor and His Virtues », Historia 30, 3, p. 298-323.

WHITBy M. (ed.) 1998, The Propaganda of Power. The Role of Panegyric in Late Antiquity, Mnemosyne. Suppl. 183, Leyde, Brill.

\section{NOTES}

1. WALLACE-HADRILL 1981; STERTZ 1977 ; SEAGER 1984 ; WHITBY (ed.) 1998 ; CHARLESWORTH 1937 ; FEARS 1981 ; CLASSEN 1991 ; NOREÑA 2001 ; GANGLOFF 2009 ; ROLLER 2001 ; EARL 1967. 
2. Sur Dion Cassius, voir MILLAR 1964 ; AALDERS 1986 ; BLOIS 1998, p. 3405 ; FREYBURGERGALLAND 1997. Sur Hérodien, voir KEMEZIS 2014 ; BLOIS 2003 ; BLOIS 1998, p. 3415. Sur Aelius Aristide, se référer à HARRIS (ed.) 2008 ; OLIVER 1980.

3. CHARLESWORTH 1937 ; BLOIS 1994, p. 166.

4. CHARLESWORTH 1937 ; PFAFF-REYDELLET 2003.

5. POTTER 1999, p. 71 ; FEARS 1981, p. 910 ; LICHOCKA 1974 ; NOREÑA 2001 ; MANDERS 2007.

6. Res Gestae Divi Augusti 34, 2 : Quo pro merito meo senatus consulto Augustus appellatus sum et laureis postes aedium mearum vestiti publice coronaque civica super ianuam meam fixa est et clupeus aureus in curia Iulia positus, quem mihi senatum populumque Romanum dare virtutis clementiaeque et iustitiae et pietatis caussa testatum est per eius clupei inscriptionem, "Pour marquer sa reconnaissance envers moi, le sénat me décerna par décret le titre d'Augustus, les montants de la porte de ma maison furent habillés de lauriers par décision officielle et une couronne civique fut accrochée au-dessus de ma porte. Un bouclier d'or fut placé dans la Curia Julia ; le sénat et le peuple romain me l'ont donné en raison de mon courage, de ma clémence, de ma justice et de ma piété ». C'est ce qu'atteste l'inscription de ce bouclier; voir GAGÉ 1932; MARKHOWSKI 1936; WALLACEHADRILL 1981, p. 300-301.

7. FEARS 1981, p. 885.

8. BLOIS 1994, p. 166 ; WALLACE-HADRILL 1981, p. 309.

9. NOREÑA 2001, p. 152 ; CHARLESWORTH 1937.

10. WALLACE-HADRILL 1981; NOREÑA 2001, p. 152-153. C'est ce que souligne POTTER 1999, p. 71 : "If [these virtues] cannot be shown to derive from some preset canon, then it follows that they were selected for advertisement because they sent a specific message about what it was that a specific emperor wanted his subjects to think about him. »

11. CLASSEN 1991, p. 38-39.

12. BIRD 1984.

13. BIRD 1984, p. 18-23. En ce qui concerne les erreurs factuelles d'Aurelius Victor, il faut se référer entre autres à son récit du règne de Pertinax (Aurelius Victor, Liber de Caesaribus, XVII, 10).

14. Aurelius Victor, Liber de Caesaribus, XIII, 8.

15. LORIOT 1990.

16. Aurelius Victor, Liber de Caesaribus, XXIX, 4.

17. RIVES 1999 ; KNIPFING 1923 ; SALISBURY, MATTINGLY 1924.

18. Aurelius Victor, Liber de Caesaribus, XXXIV, 6.

19. Voir à ce propos Ammien Marcellin, XXX, 8, 14.

20. L'austérité d'Aurélien est perçue différemment par les autres sources. Eutrope est très critique, il le qualifie de violent et de cruel (Eutrope, IX, 14) : vir in bello potens, animi tamen immodici, et ad crudelitatem propensioris. L'Histoire Auguste abonde dans le même sens en fournissant plusieurs exemples de la crudelitas d'Aurélien (SHA, Vita Aureliani, VII, 4 ; VIII, 5 ; XXXVI, 3 ; XXXIX, 8-9).

21. ALLARD 2006, p. 181-182.

22. Aurelius Victor, Liber de Caesaribus, XXXV, 7 ; voir MOLIN 2006.

23. Aurelius Victor, Liber de Caesaribus, $\mathrm{XXXV}, 6,11$. 
24. Aurelius Victor, Liber de Caesaribus, XXXV, 7 ; SHA, Vita Aureliani, XXXV, 2.

25. NOREÑA 2001, p. 160-161.

26. NOREÑA 2001, p. 160-161; VEYNE 1976, chap. 4 ; KLOFT 1970. Selon Suétone, Néron déclarait au début de son règne qu'il gouvernerait selon les préceptes de l'empereur Auguste en respectant les vertus de liberalitas, clementia, et comitas (Suétone, Nero, X, 1): Atque ut certiorem adhuc indolem ostenderet, ex Augusti praescripto imperaturum se professus, neque liberalitatis neque clementiae, ne comitatis quidem exhibendae ullam occasionem amisit.

27. Ces trois auteurs auraient utilisé une source commune: la Kaisergeschichte. L'hypothèse de l'existence de cette source commune a été émise par A. Enman, et a pour origine la similitude de leur récit. Discutée par certains spécialistes, dont P. Dufraigne, elle est aujourd'hui admise par la communauté scientifique. Voir à ce propos P. DUFRAIGNE, trad. de Aurelius Victor, Liber de Caesaribus, XXV-XXXV; BURGESS 1995 ; BIRD 1984, p. 6-37 ; BARNES 1978 ; 1968/1969, p. 22-23.

28. Aurelius Victor, Liber de Caesaribus, XXXII, 4.

29. SHA, Vita Gallieni Duo, III, 1 : turbata interim re p. toto penitus orbe terrarum, ubi Odenatus comperit Macrianum cum filio interemptum, regnare Aureolum, Gallienum remissius $<$ re>m gerere, festinavit ad alterum filium Macriani ... capiendum, «tandis que l'État était ébranlé dans le monde tout entier, lorsque Odénat apprit que Macrianus avait été tué avec son fils, qu'Auréolus régnait, que Gallien réagissait mollement, il se hâta pour capturer le second fils de Macrianus et son armée. »

30. SHA, Vita Gallieni Duo, XIV, 1.

31. Se référer à la seconde Philippique de Cicéron dans laquelle il accuse Antoine d'être efféminé et débauché. Sur cette question, voir EDWARDS 2002, p. 64-65 ; CORBEILL 1996, p. 129.

32. Il s'agit ici de traits de caractère liés à la sexualité. L'efféminé est caractérisé par son homosexualité passive. La mollesse est aussi synonyme de lubricité exacerbée, comme chez Néron (Suétone, Nero, XXVIII, 1 ; SHA, Vita Antoninus Heliogabalus, V).

33. Lorsque Galba fit défection avec les Espagnes, Néron « s'évanouit et fut longtemps le cœur abattu, sans voix et presque mort pendant ce temps-là [...], mais il n'oublia néanmoins rien de son habitude de luxe et de paresse, et ne s'en défit pas » (conlapsus animoque male facto diu sine uoce et prope intermortuus [...] nec eo setius quicquam ex consuetudine luxus atque desidiae omisit et inminuit, Suétone, Nero, XLII, 1-2). L'Histoire Auguste évoque encore la mollesse de Gallien quand elle le compare à Postumus ou Zénobie (SHA, Vita Triginta Tyranni, III, 3-5; XXX, 16).

34. La moderatio est une vertu importante pour le bon empereur. Il faut voir à ce propos les commentaires des Anciens au sujet de la moderatio de Tibère : Suétone, Tib., XXXII, 2 ; Tacite, Ann., I, 8 ; II, 36 ; III, 50 ; voir NAKAGAWA 2002. Cette absence de moderatio se manifeste par l'exubérance du caractère de l'empereur. Voir à ce propos la description d'Elagabal dans l'Histoire Auguste (SHA, Vita Antoninus Heliogabalus, V).

35. SHA, Vita Gallieni Duo, I, 1-5 ; IX, 2-3.

36. Aurelius Victor, Liber de Caesaribus, XXXIV, 4. L'Histoire Auguste va plus loin en indiquant que Claude le Gothique « exerça le pouvoir en suivant les vœux du Sénat » (SHA, Vita Divi Claudii, II, 8).

37. Aurelius Victor, Liber de Caesaribus, XXXIII, 33-34 ; LE BOHEC 2004 ; COSME 2007. 
38. Aurelius Victor, Liber de Caesaribus, XXXIII, 31.

39. CHRISTOL 2006, p. 163.

40. CHRISTOL 2006, p. 163 ; DEMOUGEOT 1969, p. 425-428.

41. Aurelius Victor, Liber de Caesaribus, XXXV, 7.

42. L'Histoire Auguste met en parallèle l'insouciance de Gallien et la sécession gauloise : SHA, Vita Gallieni Duo, IV, 3-4. Gallien abandonne l'État : SHA, Vita Gallieni Duo, XIV, 5.

43. Aurelius Victor, Liber de Caesaribus, XXXIII, 21.

44. À l'issue de la reconquête de Palmyre, Aurélien aurait effectué une brève campagne contre les Perses. L'ampleur de cette campagne reste suspecte. Il semblerait que la propagande du régime l'ait amplifiée pour justifier les titres de Persicus Maximus et de Particus Maximus. Voir à ce propos KIENAST 1990, p. 232.

45. KIENAST 1990, p. 231-232.

\section{RÉSUMÉS}

Cet article a pour objectif de présenter le modèle du bon empereur pour le $\mathrm{III}^{\mathrm{e}}$ siècle, à partir d'une analyse comparative des biographies des empereurs Gallien, Claude le Gothique et Aurélien dans les Caesares d'Aurelius Victor.

This article aims to present the model of the good emperor for the $3^{\text {rd }}$ century based on a comparative analysis of the biographies of the emperors Gallienus, Claudius II and Aurelian in the Caesares of Aurelius Victor.

\section{INDEX}

oeuvrecitee Hauts faits et gestes du divin Auguste, Histoire Auguste nomsmotscles Aurelius Victor, Cicéron, Dion Cassius, Eutrope, Hérodien, Pseudo-Aristide (Aelius), Suétone

Keywords : imperial virtues, crisis of the third century, Gallienus, Claudius II, Aurelian Mots-clés : vertus impériales, crise du troisième siècle, Gallien, Claude II, Aurélien

\section{AUTEURS}

HERMANN AMON

Université d'Ottawa 